\title{
EMPIRICAL ANALYSIS ON THE USE OF OLS IN THE ESTIMATION OF SIMULTANEOUS EQUATION MODELS
}

\section{A. AGUNBIADE}

Department of Mathematical Sciences

Olabisi Onabanjo University

Nigeria

e-mail: bayoagunbiade@gmail.com

adebayo.agunbiade@oouagoiwoye.edu.ng

\begin{abstract}
The inconsistent estimators of the parameters resulted from the simultaneity problem when the structural equations are estimated by ordinary least squares (OLS) approach is of great importance in this research. The study argues that it does not mean that OLS estimation of simultaneous equation models (SEM) is useless; and so examined the empirical analysis of the three categories of estimation techniques: OLS, single equation and system estimation approaches. The asymptotic and small sample properties of the various methods are compared using total absolute bias and the root mean square error. The findings indicated no remarkable and asymptotic pattern in the performance of the estimates of the parameters of each estimator. However, the ranking of the three categories of simultaneous estimation methods differs according to the correlation level of the explanatory variables and the individual equation of the SEM.
\end{abstract}

2010 Mathematics Subject Classification: 62Jxx.

Keywords and phrases: Monte Carlo methods, simultaneous equation models, estimating techniques, regression models, OLS approach.

Received June 30, 2017

(C) 2017 Scientific Advances Publishers 


\section{Introduction}

In many cases, a one-way or unidirectional cause-and-effect relationship between jointly dependent and some explanatory variables are required for policy formulation (Agunbiade [3]). In this regards, the simultaneous equations model (SEM) is most important to econometricians both from a theoretical as well as applied perspective. The main thrust of this study is to empirically analyse the notion on the use of OLS approach in the parameter estimation of simultaneousequation systems. Many authors like Johnston [11]; Goldberger [7]; Greene [8]; and Maddala [15] have shown that OLS cannot be applied to estimate the structural parameters of each equation in a system of simultaneous equations mainly because of the presence of current endogeneous variables in the structural equation. This research however argues that it does not absolutely imply that the application of OLS is useless and then examined and compared the three categories of estimation techniques of SEM. The obtained results were analysed by using the total absolute bias and the root mean square error RMSE. The ranking of these techniques were also made to reveal the true position of OLS. The asymptotic and small sample properties of a Monte Carlo simulation analysis were equally done.

\section{Materials and Methods}

The model to be estimated is

$$
\beta y_{t}+\Gamma x_{t}=u_{t}
$$

where $\beta$ is $G \times G$ matrix of coefficients of current values of endogeneous variables, and $\Gamma$ is $G \times K$ matrix of coefficients of pre-determined variables.

The structural form of the linear simultaneous equation model which contains $G$ equations as well as its matrix notation was well documented in Greene [8] and Johnston [11]. For clarity, the representations of the notations are: 


$$
\begin{gathered}
\beta=\left(\begin{array}{ccc}
\beta_{11} & \beta_{12} & \ldots \beta_{1 G} \\
\beta_{21} & \beta_{22} & \ldots \beta_{2 G} \\
\vdots & & \\
\beta_{G 1} & \beta_{G 2} & \ldots \beta_{G G}
\end{array}\right), \quad \Gamma=\left(\begin{array}{ccc}
\gamma_{11} & \gamma_{12} & \ldots \gamma_{1 k} \\
\gamma_{21} & \gamma_{22} & \ldots \gamma_{2 k} \\
\vdots & & \\
\gamma_{G 1} & \gamma_{G 2} & \ldots \gamma_{G k}
\end{array}\right), \\
y_{t}=\left(\begin{array}{c}
y_{1 t} \\
y_{2 t} \\
y_{G t}
\end{array}\right), \quad x_{t}=\left(\begin{array}{l}
x_{1 t} \\
x_{2 t} \\
x_{k t}
\end{array}\right), \quad U_{t}=\left(\begin{array}{c}
U_{1 t} \\
U_{2 t} \\
U_{G t}
\end{array}\right),
\end{gathered}
$$

since $t=1,2, \ldots, n$ each of the $G$ equations has $n$ forms, one for each period and hence for the $n$ periods, the model can be written as

$$
B Y+\Gamma X=U,
$$

where

$$
Y=\left(\begin{array}{lll}
y_{11} & y_{12} & y_{1 n} \\
y_{21} & y_{22} & y_{2 n} \\
y_{G 1} & y_{G 2} & y_{G n} \\
(G x n)
\end{array}\right), X=\left(\begin{array}{lll}
x_{11} & x_{12} & x_{1 n} \\
x_{21} & x_{22} & x_{2 n} \\
x_{k 1} & x_{k 2} & x_{k n} \\
(K x n) & \left(\begin{array}{lll}
u_{11} & u_{12} & u_{1 n} \\
u_{21} & u_{22} & u_{2 n} \\
u_{G 1} & u_{G 2} & u_{G n} \\
(G x n)
\end{array}\right)
\end{array}\right.
$$

The underlying assumptions about the error term as well as its implications are well treated in Kutsoyiannis [14], Johnston [11] and even discussed in Agunbiade [3]. The presentation of the system described in (2.1), (2.2), (2.3) as well the structural form of the linear simultaneous equation described in Greene [8] gave rise to our model designed $(G=3, K=3)$

$$
\begin{aligned}
& y_{1 t}=\beta_{13} y_{3 t}+\gamma_{11} x_{1 t}+\gamma_{12} x_{2 t}+u_{1 t} \\
& y_{2 t}=\beta_{21} y_{1 t}+\gamma_{21} x_{1 t}+\gamma_{23} x_{3 t}+u_{2 t} \\
& y_{3 t}=\beta_{32} y_{2 t}+\gamma_{32} x_{2 t}+\gamma_{33} x_{3 t}+u_{3 t},
\end{aligned}
$$


where

$$
\begin{gathered}
\beta=\left(\begin{array}{ccc}
1 & 0 & -\beta_{13} \\
-\beta_{21} & 1 & 0 \\
0 & -\beta_{32} & 1
\end{array}\right), \quad \Gamma=\left(\begin{array}{ccc}
-\gamma_{11} & -\gamma_{12} & 0 \\
-\gamma_{21} & 0 & -\gamma_{23} \\
0 & -\gamma_{32} & -\gamma_{33}
\end{array}\right), \\
y_{t}=\left(\begin{array}{l}
y_{1 t} \\
y_{2 t} \\
y_{3 t}
\end{array}\right), \quad x_{t}=\left(\begin{array}{l}
x_{1 t} \\
x_{2 t} \\
x_{3 t}
\end{array}\right), \quad U_{t}=\left(\begin{array}{l}
U_{1 t} \\
U_{2 t} \\
U_{3 t}
\end{array}\right) .
\end{gathered}
$$

Using Equation (2.1), the reduced form of our model is deduced as

$$
y_{t}=-\beta^{-1} \Gamma X_{t}+\beta^{-1} U_{t} .
$$

Thus, our illustrative model of (2.4) can further be simplified in the context of (2.6) as:

$$
\begin{gathered}
y_{t}=\frac{-1}{1-\beta_{13} \beta_{32} \beta_{21}}\left(\begin{array}{ccc}
1 & \beta_{13} \beta_{32} & \beta_{13} \\
\beta_{21} & 1 & \beta_{21} \beta_{13} \\
\beta_{32} \beta_{21} & \beta_{32} & 1
\end{array}\right)\left(\begin{array}{ccc}
-\gamma_{11} & -\gamma_{12} & 0 \\
-\gamma_{21} & 0 & -\gamma_{23} \\
0 & -\gamma_{32} & -\gamma_{33}
\end{array}\right)\left(\begin{array}{l}
x_{1 t} \\
x_{2 t} \\
x_{3 t}
\end{array}\right) \\
+\frac{-1}{1-\beta_{13} \beta_{32} \beta_{21}}\left(\begin{array}{ccc}
1 & \beta_{13} \beta_{32} & \beta_{13} \\
\beta_{21} & 1 & \beta_{21} \beta_{13} \\
\beta_{32} \beta_{21} & \beta_{32} & 1
\end{array}\right)\left(\begin{array}{l}
U_{1 t} \\
U_{2 t} \\
U_{3 t}
\end{array}\right)
\end{gathered}
$$

Alternatively, Equation (2.6) can be written in terms of its reduced form parameters

$$
y_{t}=\prod X_{t}+V_{t}
$$

where $\prod$ is the reduced form of parameters defined as

$$
V_{t}=\beta^{-1} u_{t} \quad \text { and } \quad \prod=-\beta^{-1} \Gamma .
$$


So,

$$
y_{t}=\left(\begin{array}{lll}
\prod_{11} & \prod_{12} & \prod_{13} \\
\prod_{21} & \prod_{22} & \prod_{23} \\
\prod_{31} & \prod_{32} & \prod_{33}
\end{array}\right)\left(\begin{array}{c}
x_{1 t} \\
x_{2 t} \\
x_{3 t}
\end{array}\right)+\left(\begin{array}{c}
V_{1 t} \\
V_{2 t} \\
v_{3 t}
\end{array}\right)
$$

Equation (2.10) is expressed

$$
\begin{aligned}
& y_{1 t}=\prod_{11} x_{1 t}+\prod_{12} x_{2 t}+\prod_{13} x_{3 t}+V_{1 t} \\
& y_{2 t}=\prod_{21} X_{1 t}+\prod_{22} x_{2 t}+\prod_{23} x_{3 t}+V_{2 t} \\
& y_{3 t}=\prod_{31} X_{1 t}+\prod_{32} x_{2 t}+\prod_{33} x_{3 t}+V_{3 t} .
\end{aligned}
$$

So,

$$
\begin{aligned}
\prod & =\frac{-1}{1-\beta_{13} \beta_{32} \beta_{21}}\left(\begin{array}{ccc}
1 & \beta_{13} \beta_{32} & \beta_{13} \\
\beta_{21} & 1 & \beta_{21} \beta_{13} \\
\beta_{32} \beta_{21} & \beta_{32} & 1
\end{array}\right)\left(\begin{array}{ccc}
\gamma_{11} & \gamma_{12} & 0 \\
\gamma_{21} & 0 & \gamma_{23} \\
0 & \gamma_{32} & \gamma_{33}
\end{array}\right) \\
& =\frac{1}{D}\left(\begin{array}{ccc}
\gamma_{11}+\beta_{13} \beta_{32} \gamma_{21} & \gamma_{12}+\beta_{13} \gamma_{32} & \beta_{13} \beta_{32} \gamma_{23}+\beta_{13} \gamma_{33} \\
\beta_{21} \gamma_{11}+\gamma_{21} & \beta_{21} \gamma_{12}+\beta_{21} \beta_{13} \gamma_{32} & \gamma_{23}+\beta_{21} \beta_{13} \gamma_{33} \\
\beta_{32} \beta_{21} \gamma_{11}+\beta_{32} \gamma_{21} & \beta_{32} \beta_{21} \gamma_{12}+\gamma_{32} & \beta_{32} \gamma_{23}+\gamma_{33}
\end{array}\right) .
\end{aligned}
$$

The other components is expressed as

$$
V_{t}=\beta^{-1} u_{t}=\frac{1}{D}\left(\begin{array}{ccc}
U_{1 t} & +\beta_{13} \beta_{32} U_{2 t} & +\beta_{13} U_{3 t} \\
\beta_{21} U_{1 t} & +U_{2 t} & +\beta_{21} \beta_{13} U_{3 t} \\
\beta_{32} \beta_{21} U_{1 t} & +\beta_{32} U_{2 t} & +U_{3 t}
\end{array}\right)
$$

Thus we have 


$$
\begin{aligned}
y_{1 t}= & \left(\frac{\gamma_{11}+\beta_{13} \beta_{32} \gamma_{21}}{D}\right) X_{1 t}+\left(\frac{\gamma_{12}+\beta_{13} \beta_{32}}{D}\right) X_{2 t}+\left(\frac{\beta_{13} \beta_{32} \gamma_{23}+\beta_{13} \gamma_{33}}{D}\right) X_{3 t} \\
& +\left(\frac{U_{1 t}+\beta_{13} \beta_{32} U_{2 t}+\beta_{13} U_{3 t}}{D}\right) ; \\
y_{2 t}= & \left(\frac{\beta_{21} \gamma_{11}+\gamma_{21}}{D}\right) X_{1 t}+\left(\frac{\beta_{21} \gamma_{12}+\beta_{21} \beta_{13} \gamma_{32}}{D}\right) X_{2 t}+\left(\frac{\gamma_{23}+\beta_{21} \beta_{13} \gamma_{33}}{D}\right) X_{3 t} \\
& +\left(\frac{\beta_{21} U_{1 t}+U_{2 t}+\beta_{21} \beta_{13} U_{3 t}}{D}\right) ; \\
y_{3 t}= & \left.\frac{\beta_{32} \beta_{21} \gamma_{11}+\beta_{32} \gamma_{21}}{D}\right) X_{1 t}+\left(\frac{\beta_{32} \beta_{21} \gamma_{12}+\gamma_{32}}{D}\right) X_{2 t}+\left(\frac{\beta_{32} \gamma_{23}+\gamma_{33}}{D}\right) X_{3 t} \\
& +\left(\frac{\beta_{32} \beta_{21} U_{1 t}+\beta_{32} U_{2 t}+U_{3 t}}{D}\right),
\end{aligned}
$$

where $D=-\left(1-\beta_{13} \beta_{32} \beta_{21}\right)^{-1}$.

In the language of Johnston [11], for the estimation of simultaneous equation models, OLS and its variants are considered to be unsatisfactory estimating techniques. He was of the opinion that the remaining endogeneous variable(s) in the model will generally be correlated with the disturbance term in the equation so that OLS will be biased and inconsistent. He however justified that only in the case of recursive models will OLS be an optimal estimating technique.

Casteneira and Nunes [5] in classifying estimating techniques of SEM also considered OLS as 'Naive method' probably because of the biased and inconsistency of OLS.

\section{Discussions}

Many authors like Johnston [11] have shown that OLS cannot be applied to estimate the structural parameters of each equation in a system of simultaneous equations. Their argument is largely based on 
the existence of current endogeneous variables in the structural equations. The identification status of the SEM as well can be used to determine the nature of estimation methods to be used. For this study, we excluded the recursive models since they are not in the category of simultaneous equation model. Since OLS method can be used in estimating the parameters sequentially. The methods of estimation of simultaneous equation system that are considered as single equation methods (GEM) which are applied to one equation at a time or complete system methods (CSM) applied to the system as a whole. Single equation methods include two-stage least squares (2SLS), indirect least squares (ILS) and limited information maximum likelihood (LIML). The systems methods are three-stage least squares (3SLS) and full-information maximum likelihood (FIML) in which all the equations of the fully specified structural model are estimated simultaneously.

\section{Single equation method (2SLS estimation method)}

\subsection{First stage regression}

Write the equation to be estimated in the form:

$$
y=\gamma_{1} \beta_{1}+X_{1} \gamma_{1}+U_{1}
$$

Regress $\gamma_{1}$ on all the pre-determined variables in the model using OLS. The objective is to purge $\gamma_{1}$ of their stochastic elements.

After regressing $\gamma_{1}$ on all the $X^{1} \mathrm{~s}$, we obtain

$$
\widehat{Y}_{1}=X\left(X_{1} X_{2}\right)^{1} X^{1} \gamma_{1}
$$

where $X=\left[X_{1} X_{2}\right]$ is the $n \times k$ matrix of all the pre-determined variables in the system.

\subsection{Second stage regression}

Regress $y$ on $\hat{Y}_{1}$ and $X_{1}$ using:

$$
y=\widehat{Y}_{1} \beta+X_{1} \gamma+U_{1} * .
$$


Note the different between (3.1) and (3.3) and between $U_{1}$ and $U_{1}^{*}$. Let $b$ and $c$ be the 2SLS estimates $\beta$ and $\gamma$, let the residuals from OLS estimate be

$$
e=-\widehat{Y} b-X_{1} c .
$$

The sum of squares of residuals is

$$
\begin{aligned}
& e^{1} e=\left(-\widehat{Y}_{1} b-X_{1} c\right)^{1}\left(y-\widehat{Y}_{1} b-X_{1} c\right) \\
&=y-y^{1} \hat{Y} b-y^{1} X_{1} c+b^{1} \widehat{Y}_{1} \hat{Y}_{1} b+b^{1} \widehat{Y}_{1}^{1} X_{1} c+c^{1} X_{1}^{1} X_{1} c \\
& \quad-b^{1} \widehat{Y}_{1}^{1}-X_{1}^{1} c^{1} y \quad+c_{1}^{1} X_{1}^{1} \widehat{Y} b \\
&=-2 y^{1} \widehat{Y}_{1} b-2 y^{1} X_{1} c+2 c^{1} X_{1}^{1} \widehat{Y}_{1} b+b^{1} \hat{Y}_{1}^{1} \widehat{Y}_{1} b+C_{1}^{1} X_{1}^{1} X_{1} c .
\end{aligned}
$$

Note that $b$ and $c$ are the only unknowns in (3.5) and their least squares estimate are obtained by minimizing (3.5) with respect to $b$ and $c$, i.e.,

$$
\begin{gathered}
\frac{\partial\left(e^{1} e\right)}{\partial b}-2 y^{1} \widehat{Y}_{1}+2 c^{1} X_{1}^{1} \widehat{Y}_{1}+2 \widehat{Y}_{1}^{1} \widehat{Y}_{1} b=0 \\
\frac{\partial\left(e^{1} e\right)}{\partial c}-2 y^{1} \widehat{X}_{1}+2 b^{1} \widehat{Y}_{1}^{1} X_{1}+2 c^{1} X_{1}^{1} X_{1}=0
\end{gathered}
$$

or

$$
\begin{aligned}
& \widehat{Y}_{1}^{1} \widehat{Y}_{1} b+\widehat{Y}_{1}^{1} X_{1} c=\widehat{Y}_{1}^{1} y \\
& \widehat{X}_{1}^{1} \widehat{Y}_{1} b+\widehat{X}_{1}^{1} X_{1} c=\widehat{X}_{1}^{1} y,
\end{aligned}
$$

or in matrix form:

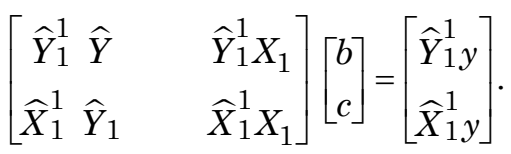


Looking at the matrix dimensions, it can win some little effort that (3.6) consists of $k+g-1$ equations in $k+g-1$ unknowns. As before each row is an equation and each column corresponds to a variable, i.e.,

$$
\left[\begin{array}{ll}
(g-1)(g-1) & (g-1) k \\
k(g-1) & k k
\end{array}\right]\left[\begin{array}{l}
(g-1) 1 \\
k .1
\end{array}\right]=\left[\begin{array}{l}
(g-1) \cdot 1 \\
k .1
\end{array}\right]
$$

states the dimensions of the eight components of (3.7) in practice, we do

not have to compute $\hat{Y}_{1}$ and it can be shown after some efforts involving the eliminate of $\widehat{Y}_{1}$ that (3.6) can be stated as:

$$
\left[\begin{array}{ll}
Y_{1}^{1} X\left(X^{1} X\right)^{-1} X^{1} Y_{1} & Y_{1}^{1} X_{1}^{1} \\
X_{1}^{1} Y_{1} & X_{1}^{1} X_{1}
\end{array}\right]\left[\begin{array}{l}
b \\
c
\end{array}\right]=\left[\begin{array}{c}
Y_{1}^{1} X\left(X^{1} X\right)^{-1} X^{1} Y \\
X_{1}^{1} y
\end{array}\right] .
$$

It can be shown that 2 SLS estimators are consistent, i.e.,

$$
\operatorname{plim}\left[\begin{array}{l}
b \\
c
\end{array}\right]=\left[\begin{array}{l}
\beta \\
\gamma
\end{array}\right]
$$

\section{Complete systems method (3SLS estimation method)}

We have discussed single-equation method of estimating the parameters of simultaneous equation models with a particular reference to a 2SLS. The inconsistency of OLS in this context was noted. Johnston [11] emphasised identification criteria as one to show that equations that are exactly identified can be estimated using the reduced form or ILS estimation method. Indeed, it should be noted that 2SLS can also be used in estimating the parameters of such an equation. When an equation is over-identified, its structural parameters can be estimated by using the 2SLS estimation method; LIML method can also be used. Hence, our emphasis on 2SLS as an example of GEM to buttress its importance. The 3 -stage least squares (3SLS) is a systems version of 2SLS and can also be used to estimate simultaneously all the parameters of a simultaneous equation model provided no equation is unidentified. However, it requires that all equations be correctly specified since one incorrectly 
specified equation will adversely affect the entire system. A systems method is applied to all the equations of the model at the same time and gives estimates of all the parameters simultaneously. It takes into account the entire structure of the model with all the restrictions that this structure imposes on the values of the parameters. Three-stage least squares is a straightforward extension of 2SLS, and involves the application of least squares in three stages. The first two stages are the same as 2SLS (as discussed above) except that we deal with the reduced form of all the equations of the system. The third stage involves the application of generalized least squares, that is, the application of least squares to a set of transformed equation in which the transformation required is obtained from the reduced-form residuals of the previous stage.

\section{Concluding Remarks}

It has been argued that OLS cannot be applied to estimate the structural parameters of each equation in a system of simultaneous largely because of the presence of current endogeneous variables in the structural equations. This study has proven that OLS estimation of SEM may not be totally useless. That the estimation techniques of SEM will differs in ranking according to the correlation level of the explanatory variables and the identification status of the individual equation of SEM.

\section{References}

[1] D. A. Agunbiade and N. O Adeboye, Estimation of heteroscedasticity effects in a classical linear regression model of a cross-sectional data, Progress in Applied Mathematics 4(2) (2012), 18-28. www.cscanada.net

[2] D. A. Agunbiade and J. A. Osilagun, Identifiability criteria in a simultaneous equation model, African Journal of Pure Applied Sciences 1(1) (2008), 34-38.

[3] D. A. Agunbiade, Parameters estimation of simultaneous equations model in the presence of multicollinearity, Bull. Cal. Math. Soc. 103(6) (2011), 489-500.

[4] D. A. Agunbiade and J. O. Iyaniwura, Estimation under multicollinearity: A comparative approach using Monte Carlo methods, Journal of Mathematics and Statistics 6(2) (2010), 183-192. www.scipub.org 
[5] B. R. Casteneira and L. C. Nunes, Testing Endogeneity in a Regression Model: An Application of Instrumental Variable Estimation, Investigation Operative 8 (1, 2 and 3) (1999),

[6] R. C. Fair, The estimation of simultaneous equation models with lagged endogenous variables and first order serially correlated errors, Econometrica 38(3) (1970).

[7] A. S. Goldberger, A Course in Econometrics. Harvard University Press, 1991.

[8] William H. Greene, Econometric Analysis, Fifth Edition, Upper Saddle River, New Jersey, Prentice Hall, 2003.

[9] D. N. Gujarati and Sangeetha, Basic Econometrics, Tata McGraw-Hill Edition (Fourth Edition) (2007), 1036. ISBN 978-0-07-066005-2

[10] M. Intriligator, R. Bodkin and C. Hsiao, Econometric Models Techniques and Applications, Prentice Hall, International Edition, 1996.

[11] J. Johnston, Econometrics Methods, Fifth Edition, McGraw - Hill Book Company, New York, 2007.

[12] J. Kmenta, Element of Econometrics, Macmillan, New York, 1971.

[13] A. Korostelev and O. Korosteleva, Mathematical statistics, Asymptotic minimax theory, Applied Mathematics of American Mathematical Society 119 (2011).

[14] A. Kutsoyiannis, Theory of Econometrics: An Introductory Exposition of Econometric Methods, Second Edition, Palgrave, New York, 2003.

[15] G. S. Maddala, Introduction to Econometrics, Third Edition, John Wiley and Sons Ltd., (2005), 636. ISBN 9971-51-383-8.

[16] S. K. Mishra, Estimation under Multicollinearity Application of Restricted Liu and Maximum Entropy Estimators to the Portland Cement Dataset, 2004. http://mpra.ub.uni-muenchen.de/1809/

[17] D. C. Montgomery, E. A. Pack and G. G. Vining, Introduction to Linear Regression Analysis, Third Edition, Wiley Series in Probability and Statistics, (2007), 641. ISBN 81-265-1047-1

[18] A. L. Nagar, A Monte Carlo study of alternative simultaneous equation estimators Econometrica 28(3) (1969), 573-590.

[19] O. E. Oduntan, The problem of multicollinearity phenomenon in a simultaneous equation. A Monte Carlo Approach. An unpublished PhD. Thesis submitted to the Department of Statistics, University of Ibadan, Ibadan, 2005.

[20] J. K. Olayemi and S. O. Olayide, Elements of Applied Econometrics (1998).

[21] J. B. Parker, The use of the Monte Carlo methods for solving large scale problems in neutronics, Journal of Royal Statistical Society of America 135(1) (1972), 16-37.

[22] H. M. Wagnar, A Monte Carlo study of estimates of simultaneous linear structural Equations, Econometrica 26 (1958), 117-133. 